\title{
Robust Watermarking of Document and Graphics Images in Wavelet Domain
}

\author{
C. Patvardhan \\ Electrical Engg.Deptt. \\ Dayalbagh Educational Institute \\ Dayalbagh, Agra, UP, India
}

\author{
A. K. Verma \\ Electrical Engg.Deptt. \\ Dayalbagh Educational Institute \\ Dayalbagh, Agra, UP, India
}

\author{
C. Vasantha Lakshmi \\ Physics \& Computer Sc.Deptt. \\ Dayalbagh Educational Institute \\ Dayalbagh, Agra, UP, India
}

\begin{abstract}
In this paper, a wavelet based watermarking scheme for the protection of document and graphics images is proposed. Document and graphics images are different from other images as such images are typically in two colors only (generally white as background and black or other color as text or drawing). In document or graphics images, white background area is usually more than the text or drawing content, therefore any attempt at watermarking, creates the problem of visual artifacts on white background. Here a spread spectrum watermarking scheme in wavelet domain is presented, which utilizes only the area covered by the text or drawing for watermarking and leaving the white background area clear of any visual artifacts. Suitable wavelet coefficients are determined for watermark embedding using a mask. Depending on the number of available wavelet coefficients for embedding, better robustness is achieved by selecting appropriate embedding strength using a simple fuzzy rule. This scheme utilizes the characteristics of human visual system by selecting high frequency wavelet coefficients of diagonal orientation. As can be seen in the computational results, clear white background area is ensured with better robustness presented on a variety of document and graphics images.
\end{abstract}

\section{General Terms}

Digital Media Security, Digital Image Watermarking.

\section{Keywords}

Wavelets; Bior6.8; Robustness; Correlation; Fuzzy Logic; Embedding Strength; Level of Decomposition; Human Visual System.

\section{INTRODUCTION}

Digital watermarking of multimedia content has become an active field of research and development. The swift advancements in the area of internet and computer technology have enabled authorized as well as unauthorized manipulation and imitation of digital multimedia products. Therefore, design and development of effective digital multimedia protection methods to prevent this have become essential in present time.

To achieve protection, a watermark is permanently embedded in the digital data to identify its origin. An efficient and effective watermarking technique has to satisfy at-least three major requirements i.e. Imperceptibility, Robustness and amount of Payload. Simultaneously maximizing all these three parameters together is difficult as they are non-commensurable. Therefore, finding an optimal solution to the problem of watermarking is a challenging task.
Digital watermarking algorithms can be classified into two main categories based on watermark embedding domain, i.e. spatial domain or frequency domain. Earlier techniques of watermark embedding utilized the spatial domain by modifying least significant bit (LSB) of an image [1]. However such techniques have relatively low-bit capacity and are not robust against various attacks such as JPEG compression, cropping and noise addition. Some improvements in spatial domain techniques are presented in $[2,3]$. Frequency domain based techniques provide better imperceptibility and robustness to a variety of attacks. Various types of frequency transforms that can be used are Discrete Fourier Transform (DFT), Discrete Cosine Transform (DCT), and Discrete Wavelet Transform (DWT). Earlier watermarking schemes used DFT $[4,5]$ and DCT $[6,7,8]$. However, Wavelet based watermarking schemes are more attractive. A good comparison of all these transform domain techniques is presented in [9]. Wavelet based watermarking methods exploit the frequency information and spatial information of the transformed data in multiple resolutions to gain robustness. A comparative analysis of performance of various wavelets is presented in [10]. Some wavelet based watermarking methods are reported in [11, 12].

The document and graphics images differ from ordinary images. These images generally are of two colors for example white for background and black for text or drawing. Such images carry a large portion of white background and small content of either text or graphics. Therefore, the normal methods of image watermarking may create visual artifacts which may be clearly visible in large background areas degrading image visual quality. To watermark such images a different approach is needed. Earlier attempts to watermark document images are presented in [13, 14]. These techniques are spatial domain approaches and focused in form of line shift, word shift and slight modifications to the characters also. A method for data hiding in binary text documents by embedding data in the 8 connected boundary of a character is given in [15]. Another method of data hiding for text document images based on pixel flipping based on some connectivity in local neighborhood of pixel is proposed in [16]. A method for Chinese characters on the basis of inter character spacing is presented in [17]. Most of the methods proposed are in spatial domain considering binary images but practically, text or drawing images are not exact binary images. A text document or drawing, when converted electronically, it rendered as either a color ( 24 bit) image or a grayscale ( 8 bit) image preserving smoothness of character boundary or drawing. When such images are converted into binary, the smoothness is lost and spoils the document image with broken characters and irregular boundaries. The drawing 
images having fine details may become useless in some sense after binarization. A method based on edge histogram for grayscale images is proposed in [18]. Some comparisons and recent developments in area of document image watermarking are reported in $[19,20]$.

A few efforts have been made in transform domain for watermarking of such images recently. A DCT based method for embedding watermarks in the DC components of DCT of binary images using a biased binarization threshold is presented in [21]. A method using wavelet transform is proposed in [22], where, watermark is embedded in lowest frequency sub bands along with Krawtchouk moment for better robustness. Another method employing fractal coding for Arabic and Farsi characters is presented in [23]. Most of the these methods are mainly for the problem of watermarking of text documents focusing on pixel flipping, changing interline or inter character space or introducing slight modification to character shapes and thus are not suitable for drawing images. In this paper, a new method is presented which utilizes the advantages of wavelet transform as well robustness of spread spectrum watermarking. Results show that the proposed method is well suitable for text documents as well as graphics images.

The rest of the paper is organized as follows. Section 2 provides a brief introduction to wavelet transforms analysis. Section 3 describes the proposed watermark embedding and watermark detection algorithms. Section 4 presents the experimental results. Some conclusions are given in section 5 .

\section{DISCRETE WAVELET TRANSFORM AND FILTER BANKS}

Wavelet transform plays an important role in the area of signal and image processing. Wavelet transform uses inner products to measure the similarity between a signal and an analyzing function. Here analyzing function is wavelet $\psi(t)$. To compute wavelet transform, the signal or image is compared with shifted and compressed or stretched versions of a wavelet function. Stretching or compressing a wavelet function is collectively referred to as dilation or scaling and shifting is known as translation. If scales and positions are chosen based on powers of two, so-called dyadic scales and positions, then analysis becomes much more efficient and just as accurate. It was developed in 1988 by $\mathrm{S}$. Mallat. The discrete form of wavelet $\psi_{m, n}(k)$ constitutes an orthonormal basis for $\mathbb{L}^{2}(\mathbb{R})$ and is represented as,

$$
\psi_{m, n}(k)=2^{-\frac{m}{2}} \psi\left(2^{-m} k-n\right) m, n \in \mathbb{Z}
$$

For a given function $f(k)$, the inner product $\left\langle f, \psi_{m, n}\right\rangle$ then gives the discrete wavelet transform defined as, [24]

$$
D W T(m, n)=\left\langle f, \psi_{m, n}\right\rangle=2^{-\frac{m}{2}} \sum_{k=-\infty}^{\infty} f(k) \cdot \psi^{*}\left(2^{-m} k-n\right)
$$

Wavelet orthonormal bases of images can be constructed from wavelet orthonormal basis of one dimensional signal. Three mother wavelets $\psi^{1}(x), \psi^{2}(x)$ and $\psi^{3}(x)$ with $x=\left(x_{1}, x_{2}\right) \in$ $\mathbb{R}^{2}$, are dilated by $2^{j}$ and translated by $2^{j} n$ with $n=\left(n_{1}, n_{2}\right) \in$ $\mathbb{Z}^{2}$. This yields an orthonormal basis of the space $\mathbb{L}^{2}\left(\mathbb{R}^{2}\right)$ of finite energy functions $f(x)=f\left(x_{1}, x_{2}\right)$ :

$$
\left\{\psi_{j, n}^{k}(x)=\frac{1}{2^{j}} \psi^{k}\left(\frac{x-2^{j} n}{2-j}\right)\right\}_{j \in Z, n \in Z^{2}, 1 \leq k \leq 3}
$$

The support of a wavelet $\psi_{j, n}^{k}$ is a square of width proportional to the scale $2^{j}$. Two dimensional wavelet bases are discretized to define orthonormal bases of images including $N$ pixels. Wavelet coefficients are calculated with the fast $O(N)$ algorithm using multirate filter banks [25]

The wavelet decomposition of an image based on the multi resolution theory can done using digital FIR filters [26] as shown in figure 1 .

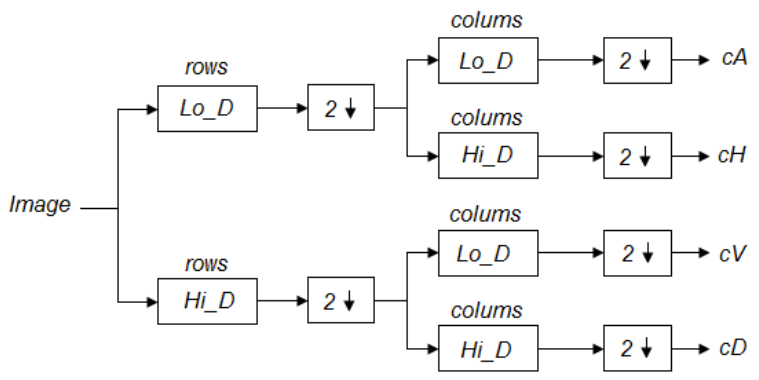

Figure1: One level wave let decomposition of an image

In the figure 1, Lo_D represents a Low Pass FIR filter and Hi_D represents a High Pass FIR filter. The input image of size $M \times M$ is converted into four coefficients matrices $\mathrm{cA}, \mathrm{cH}, \mathrm{cV}$ and $\mathrm{cD}$ of $\operatorname{size} \frac{M}{2} \times \frac{M}{2}$. The coefficients represented by $\mathrm{cA}$ are called approximation coefficients and contain low frequency details of the image while coefficients $\mathrm{cH}, \mathrm{cV}$ and $\mathrm{cD}$ are called Detailed coefficients and contain horizontal, vertical and diagonal high frequency details of the image. The wavelet decomposed image can be reconstructed back by these coefficients using Inverse DWT as shown in figure 2.

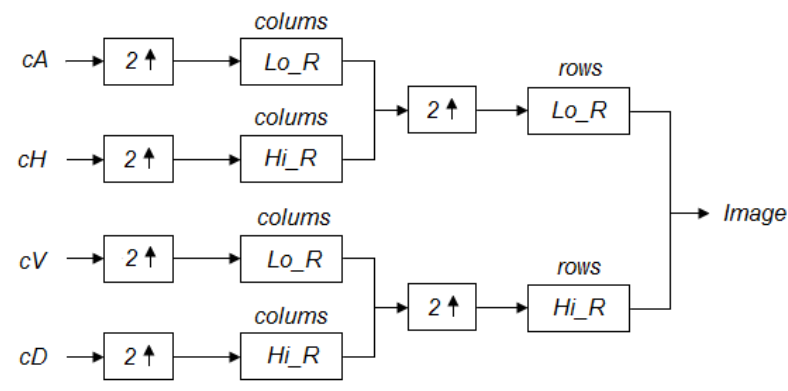

Figure 2: One level wave let Reconstruction of an Image

Figures 1 and 2 show one level wavelet-based decomposition and reconstruction. Multi-level decomposition can be achieved by further decomposing approximation coefficient matrix $\mathrm{cA}$ similar to the scheme of figure 1. Development of many wavelets is motivated by improvements on their previous versions or to fulfill the particular requirement of a specific application. Accordingly, wavelets such as Haar, Db, Bior, Symlet, Coiflet and the newer Ridge lets, Contour lets, Curvelets and Shearlets etc. are in common use.

In the scheme proposed, higher order Bi-Orthogonal wavelet 'Bior6.8' is used due to its several advantages such as perfect reconstruction and smoothness. Another advantageous property of biorthogonal over orthogonal wavelets is that they have higher embedding capacity if they are used to decompose the image into different channels [27]. 


\section{WATERMARK EMBEDDING AND EXTRACTION ALGORITHM}

In this paper, a version of the spread spectrum watermarking scheme [28] is implemented for the proposed robust algorithm. The watermarking is achieved by adding a Pseudo Random Noise (PN) sequence to the selected high frequency diagonal wavelet coefficients for each of the watermark bit and watermark is extracted by finding correlation between regenerated PN sequence and modified wavelet coefficients. The selection of appropriate wavelet coefficients for watermarking is done by a mask and embedding strength is obtained by fuzzy logic, based on number of selected wavelet coefficients. The overall system of watermarking and watermark extraction is shown in figure 3 .

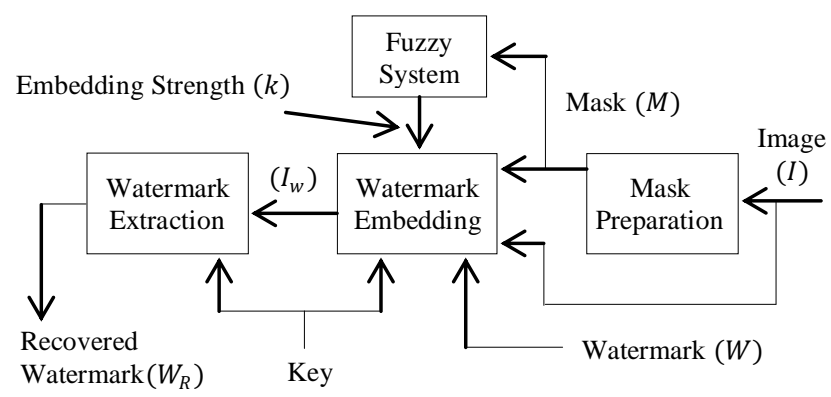

Figure 3: Proposed Watermarking Scheme

\subsection{Mask Preparation}

Document and graphics images have large white background; therefore, unless proper care is taken in embedding, artifacts are visible clearly in white areas even though high PSNR may be achieved. Although such effect can be minimized by keeping embedding strength low, but this causes poor robustness. Here an attempt has been made to select only those wavelet coefficients for modification, which are the part of image content i.e. either on the text area or drawing area only. No coefficient representing white background is altered. To get such wavelet coefficients, a mask is prepared as shown in figure 4 .

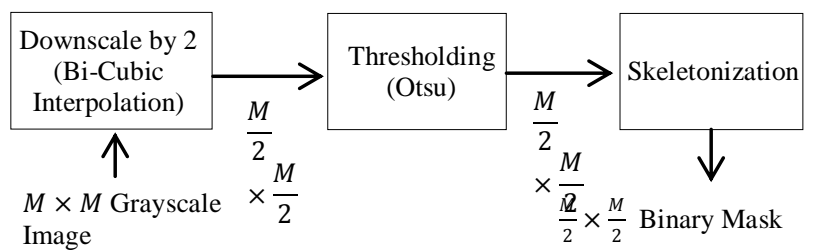

Figure 4: Mask Preparation Process

In this process, Firstly grayscale image of size $M \times M$ is downscaled by 2 preferably using cubic interpolation to get $\frac{M}{2} \times \frac{M}{2}$ size image. Then it is converted into binary image by thresholding using Otsu's method. This binary image is then skeletonized to get fine lines representing text or drawing area. Proper skeletonization is required to get fine lines to select wavelet coefficients to be modified otherwise rough binary mask can select wavelet coefficients outside the contents in white background area. One example image and its corresponding mask (inverted and enlarged) are shown in figure 5.

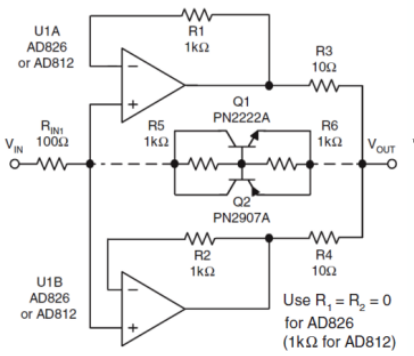

Dual op amp buffer circuit

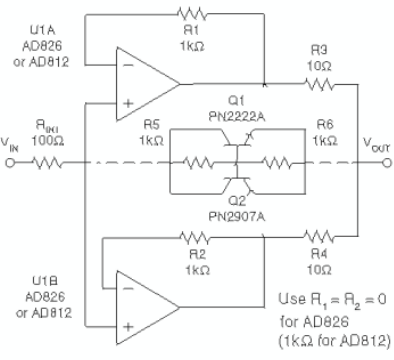

Dual op amp buffer circuit
Figure 5: Example Image and its corresponding mask

\subsection{Fuzzy based Embedding Strength Selection}

The mask obtained as shown in section 3.1 is used to select wavelet coefficients for watermark embedding simply by multiplying it with frequency band to be modified (in figure 5 , an inverted mask is shown for better print purpose). It is evident from the figure 5 , that number of pixels representing image content or detail is much lesser than white background pixels. In watermarking techniques in literature, pseudo random sequence is embedded in whole frequency band. In case of such images, the number of available coefficients is very less to modify and this number of available coefficients will vary depending on image contents. Lesser the available coefficients poorer is the robustness. Therefore, to achieve same level of robustness, the embedding strength has to be adjusted depending on image contents. In case of lesser available coefficients, the embedding strength ' $k$ ' has to be increased and can be decreased when number of available coefficients are large for modification. For this purpose a fuzzy system is used to take decision about embedding strength depending on image contents. The ratio of available coefficients to total number of coefficients in frequency band of interest or ratio of black pixels to total number of pixels in mask is taken as input to the fuzzy system to take decision for appropriate embedding strength. This ratio is represented by,

$$
\text { bwratio }=\frac{\text { total black pixels in mask }}{\text { total no.of pixels in mask }}
$$

For document and graphics images, it is found that this ratio typically ranges from 0.03 to 0.2 . If bwratio goes above 0.18 then traditional methods of watermarking can be applied and if bwratio goes below 0.05 then the proposed method can create visual artifacts in black areas of image due to high value of embedding strength ' $k$ '. In the proposed scheme the range of bwratio is taken from 0.05 to 0.18 and corresponding range of embedding strength ' $k$ ' is found empirically to be 2 to 8 for various test images to keep the robustness up to a certain desirable level in terms of mean correlation of pseudo random noise and modified frequency band. A simple fuzzy system is defined to take bwratio as input and give embedding strength ' $k$ ' as output. The block diagram and corresponding membership functions with range are shown in figure $6(\mathrm{a}), 6$ (b) and 6 (c). 


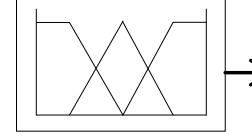

bwratio

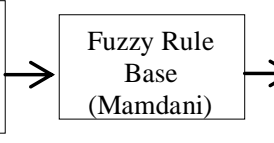

(a)

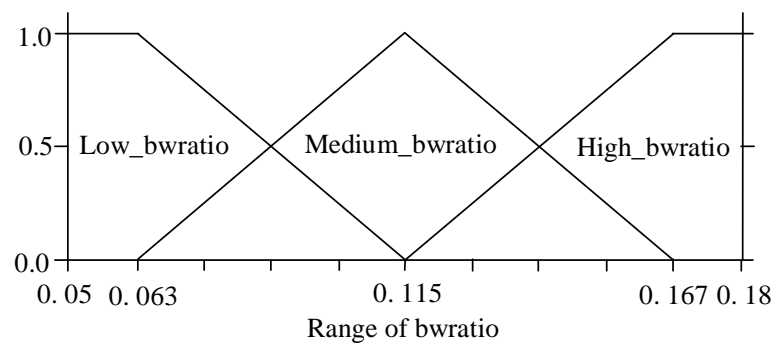

(b)

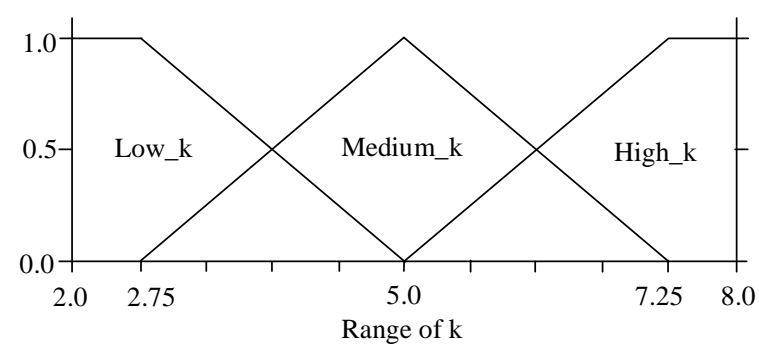

(c)

Figure 6: (a) Fuzzy system, (b) Input membership functions, (c) Output membership functions

In the fuzzy system shown in figure 6, the fuzzy model used is Mamdani and defuzzification method used is Centroid. The following fuzzy rules are used in rule base,

i) If input is (low_bwratio) then output is (high_k)

ii) If input is (medium_bwratio) then output is (medium_k)

iii) If input is (high_bwratio) then output is (low_k)

\subsection{Watermark Embedding Algorithm}

The steps of embedding algorithm are as follows:

Input: A grey scale image $(I)$ of type uint 8 and of size $M \times$ $M$ and a binary watermark $(W)$.

Output: Watermarked Image $\left(I_{w}\right)$.

1) Do the one level wavelet decomposition of Host Image (I) obtaining four coefficients matrices $c A, c H, c V$ and $c D$ of $\operatorname{size} \frac{M}{2} \times \frac{M}{2}$.

2) The high frequency band $c D$ is selected for watermark embedding and a binary Mask $(M)$ is prepared as per the procedure of section 3.1.

3) Select a seed to generate a pseudo random sequence $(P N S)$ of size equal to the size of frequency band $c D$. Modify $P N S$ to get another sequence $(P N)$, which contains only $+1,-1$ and 0 according to the equation $P N=R 1 \times$ $(P N S-R 2)$. Where, $R 1=2$ and $R 2=0.5$.

4) Convert the $2 \mathrm{D}$ watermark into $1 \mathrm{D}$ array and if watermark bit is 0 (Black) then modify the $c D$ wavelet coefficients as,

$$
c D^{\prime}=c D+k \cdot(M \times P N)
$$

Where, $k$ is embedding strength and $M$ is the mask. If watermark bit is 1 (white) then wavelet coefficients are left unchanged.

5) Repeat the steps 3 and 4 for all ' 0 ' watermark bits with every time newly generated $\mathrm{PN}$ sequence each time.

6) Take modified $c D^{\prime}$ to its original position and take inverse DWT to get back the watermarked image $\left(I_{w}\right)$.

7) Compute the PSNR for Iand $I_{w}$ to check that how much the host image is modified.

The embedding algorithm is shown below graphically in figure 7.

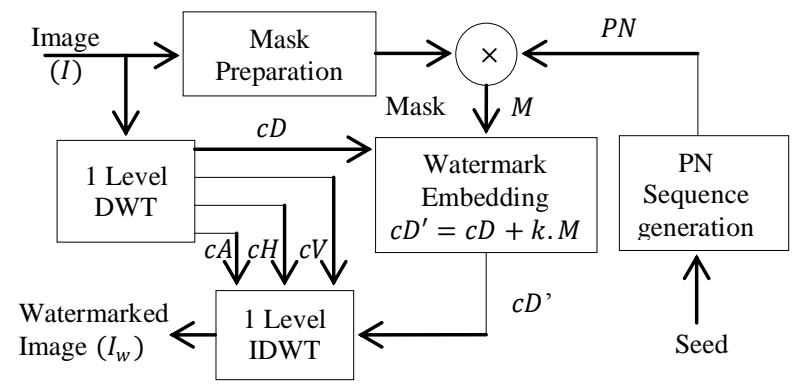

Figure 7: Watermark Embedding Algorithm

\subsection{Watermark Extraction Algorithm}

Following are the steps in watermark extraction,

Input: Watermarked Image $\left(I_{w}\right)$.

Output: Extracted Watermark $\left(W_{R}\right)$.

1) Decompose the watermarked image $\left(I_{w}\right)$ in 1 level wavelet coefficient metrics and get $c D^{\prime}$.

2) Generate the same pseudo random sequence $(P N S)$, which was generated in embedding process using same Seed value and convert this sequence (PNS) into $P N$ as $P N=R 1 \times$ $(P N S-R 2)$ with $R 1=2$ and $R 2=0.5$.

3) Compute the correlation coefficients between pseudo random sequence $(P N)$ and modified coefficient metrics $c D^{\prime}$ selected by the mask $M$ as, $r=\operatorname{corr} 2((P N *$ $M),\left(C D^{\prime} * M\right)$, Where $M$ is mask. Repeat steps 2 and 3 for all watermark bits and compute all correlation values $r$.

4) Compute the Threshold value as $(T=1.5 *$ mean $(r))$ and initialize a row matrix $\left(W^{\prime}\right)$ having all values ' 1 ' equivalent to the size of watermark.

5) For every watermark bit, compare $r$ with $T$ and modify the $W^{\prime}$ as follows,

$$
W^{\prime}= \begin{cases}0, & r>T \\ 1, & \text { otherwise }\end{cases}
$$

6) Reshape the row matrix $W^{\prime}$ into a matrix equivalent to the size of original watermark matrix $(W)$ to get recovered watermark $\left(W_{R}\right)$.

7) Compute the correlation between original watermark $(W)$ and recovered watermark $\left(W_{R}\right)$ to check the quality of recovered watermark.

The watermark extraction algorithm is shown below graphically in figure 8 .

\section{EXPERIMENTAL RESULTS AND ANALYSIS}

This section presents the experimental results. As host images four grayscale images of size $512 \times 512$ are tested. These images are shown in figure 9. 


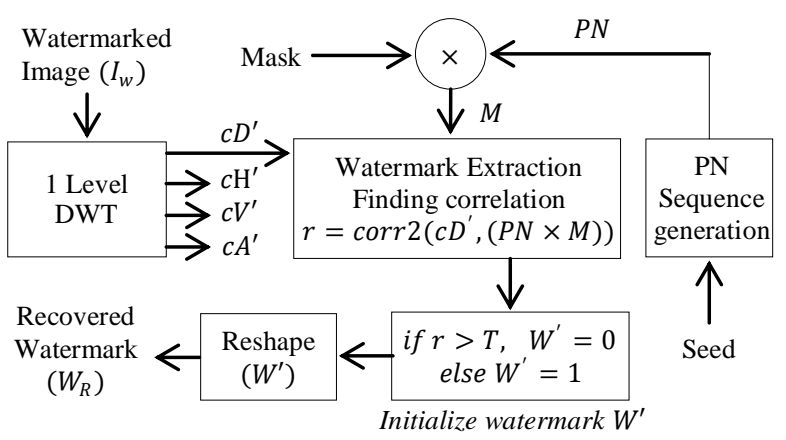

Figure 8: Watermark Extraction Algorithm

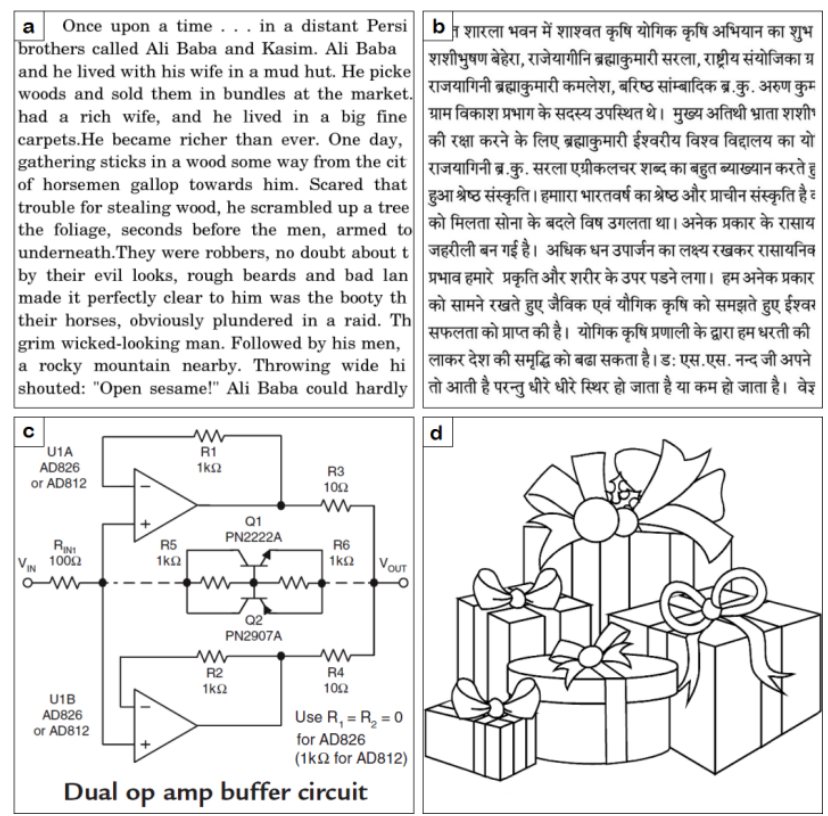

Figure 9: Various Test Images: (a) English Text (b) Hindi Text (c) Electronic Circuit (d) Drawing Image

The watermark image taken is a binary image of size $21 \times 12$ as shown in figure 10 .

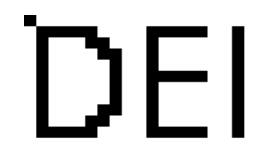

Figure 10: Binary Watermark Image

The binary watermark as shown in figure 10 is embedded in diagonal frequency band of host image using the embedding algorithm of section 3.3. After watermark embedding, PSNR between original image $(I)$ and watermarked image $\left(I_{w}\right)$ is calculated using,

$$
P S N R=10 \log _{10}\left(\frac{255^{2}}{M S E}\right)
$$

Where, MSE is mean square error given by,

$$
M S E=\frac{1}{N^{2}} \sum_{i=1}^{N} \sum_{i=1}^{N}\left[I(i, j)-I_{w}(i, j)\right]^{2}
$$

Where, $I(i, j)$ and $I_{w}(i, j)$ represent pixels of original image and watermarked image respectively at location $(i, j)$. For the test images of figure 9, PSNR value after watermarking, their bwratio and corresponding embedding strength ' $k$ ' is shown in the table 1 .

Table1: Comparison of PSNR, bw ratio and embedding strength ' $k$ '

\begin{tabular}{|c|c|c|c|}
\hline Images & PSNR (dB) & bwratio & $\mathrm{K}$ \\
\hline Image_a & 35.7830 & 0.1187 & 4.9408 \\
Image_b & 36.1778 & 0.1498 & 4.0072 \\
Image_c & 36.1000 & 0.0555 & 6.9668 \\
Image_d & 35.1983 & 0.0645 & 6.8580 \\
\hline
\end{tabular}

As shown in table 1 , as bwratio decreases, the value of ' $k$ ' increases to maintain the robustness at a certain desirable level. The watermarked images are shown in figure 11 .

From the zoomed part of figure 11, the quality of watermarking is observed as no visual artifacts are visible in the image. The performance of proposed watermarking scheme is tested under several attack cases such as compression, noise addition, filtering and geometric distortions. The quality of extracted watermark is judged by finding Normalized Correlation Coefficient (NC) between original watermark $(W)$ and recovered watermark $\left(W_{R}\right)$ by the relation,

$$
N C=\frac{\sum_{i} \sum_{j} W(i, j) \cdot W_{R}(i, j)}{\sqrt{\sum_{i} \sum_{j} W^{2}(i, j)} \cdot \sqrt{\sum_{i} \sum_{j} W_{R}^{2}(i, j)}}
$$

Performance of the proposed scheme under various attack cases is investigated in the rest of the section.

The robustness of proposed scheme is tested against JPEG compression. The results are shown in table 2. As seen from the results, the watermark is able to well survive upto quality factor 30 or even below this.

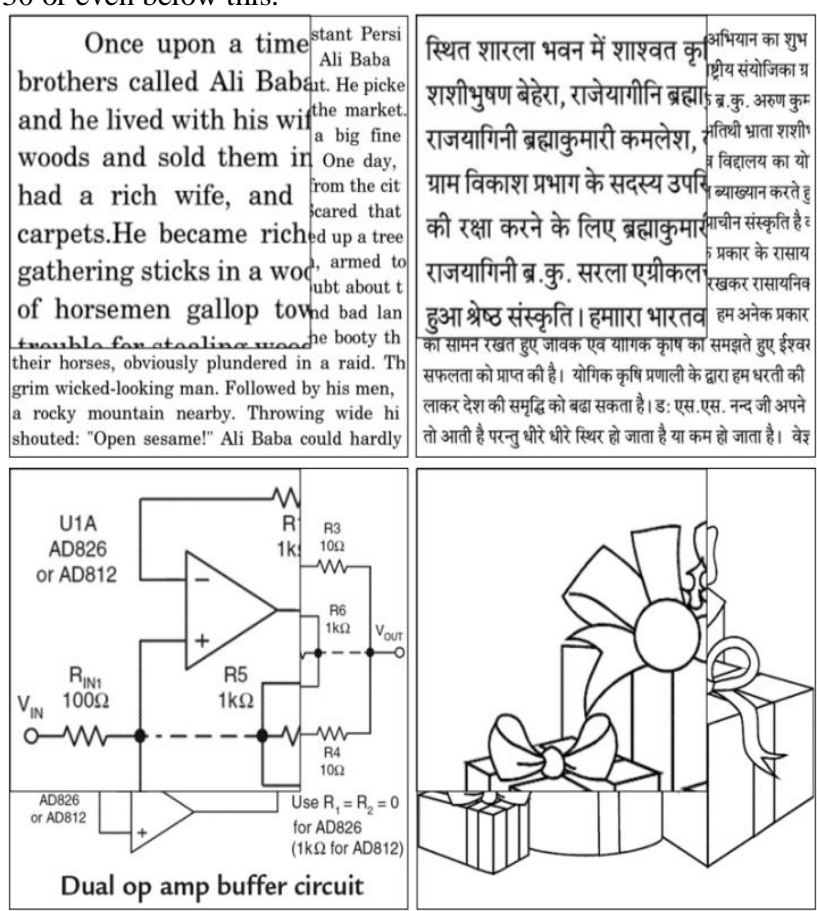

Figure 11: Watermarked Images (Zoomed part at left upper corner) 
Table 2: Values of NC under JPEG compression (Quality

\begin{tabular}{|c|c|c|c|}
\multicolumn{5}{c|}{ Factor $=\mathbf{7 0}, \mathbf{5 0}, \mathbf{3 0}$ ) attack } \\
\hline Images & $\mathrm{Q}=70$ & $\mathrm{Q}=50$ & $\mathrm{Q}=30$ \\
\hline Image_a & 1.0000 & 0.9050 & 0.7800 \\
Image_b & 1.0000 & 0.9380 & 0.7680 \\
Image_c & 1.0000 & 0.9480 & 0.7203 \\
Image_d & 0.9891 & 0.9480 & 0.7019 \\
\hline
\end{tabular}

In case of impulsive noise such as Salt ' $n$ ' Pepper noise, the performance of proposed scheme is excellent as evident from table 3. As seen from the results, the value of normalized correlation coefficient between original and recovered watermark for various noise strengths of Salt ' $n$ ' Pepper noise is very close to 1 .

Table 3: Values of NC under Salt \& Pepper Noise attack (Strength $(S)=0.02,0.04,0.06)$

\begin{tabular}{|c|c|c|c|}
\hline Images & $\mathrm{S}=0.02$ & $\mathrm{~S}=0.04$ & $\mathrm{~S}=0.06$ \\
\hline Image_a & 1.0000 & 0.9570 & 0.9681 \\
Image_b & 1.0000 & 1.0000 & 0.9785 \\
Image_c & 1.0000 & 0.9891 & 0.9785 \\
Image_d & 1.0000 & 0.9681 & 0.9579 \\
\hline
\end{tabular}

It also performs fairly well, in case of Gaussian noise attack. As given in table 4 , for Gaussian noise of zero mean $(\mu=0)$ and different variance $\left(\sigma^{2}\right)$ values $(0.010,0.015,0.02)$, the value of $\mathrm{NC}$ is near to 1 .

Table 4: Values of NC under Gaussian Noise attack $(\mu=0$

\begin{tabular}{|c|c|c|c|}
\hline and $\boldsymbol{\sigma 2}=\mathbf{0 . 0 1 0}, \mathbf{0 . 0 1 5}, \mathbf{0 . 0 2})$ \\
\hline Images & $\sigma^{2}=0.010$ & $\sigma^{2}=0.015$ & $\sigma^{2}=0.020$ \\
\hline Image_a & 1.0000 & 0.9891 & 0.9891 \\
Image_b & 1.0000 & 1.0000 & 1.0000 \\
Image_c & 1.0000 & 0.9785 & 0.9681 \\
& 1.0000 & 1.0000 & 0.9781 \\
\hline
\end{tabular}

The proposed scheme also shows its robustness against cropping. As can been seen from table 5, the watermark well survives even in the case of half image is cut and value of $\mathrm{NC}$ is nearly ' 1 '. In the table 5 , two cases are taken. In first case, quarter part of image is cut from top left corner and in second case; the half upper part of host image is cut. In case of cropping beyond this, the embedded watermark survives excellently well.

Table 5: Values of NC under Cropping attack (1/4th upper left corner is cut and Upper half is cut)

\begin{tabular}{|c|c|c|}
\hline \multicolumn{3}{|c|}{ left corner is cut and Upper half is cut) } \\
\hline Images & $\begin{array}{c}\text { Upper left } 1 / 4^{\text {th }} \\
\text { part is cut }\end{array}$ & Upper half is cut \\
\hline Image_a & 1.0000 & 1.0000 \\
Image_b & 1.0000 & 1.0000 \\
Image_c & 0.9891 & 0.9681 \\
Image_d & 1.0000 & 0.9892 \\
\hline
\end{tabular}

In case of intensity sharpening and histogram equalization, the robustness of proposed scheme is evident from table 6 and table 7 respectively.

Table 6: Values of NC under Sharpening attack (Mask $=[-1$

\begin{tabular}{|l|c|}
\hline \multicolumn{2}{|c|}{$\mathbf{- 1} \mathbf{- 1} \mathbf{- 1} \mathbf{9 - 1} \mathbf{- 1} \mathbf{- 1} \mathbf{- 1}])$} \\
\hline Images & NC \\
\hline Image_a & 0.9681 \\
Image_b & 1.0000 \\
Image_c & 0.9785 \\
Image_d & 0.9786 \\
\hline
\end{tabular}

Table 7: Values of NC under Histogram Equalization attack

\begin{tabular}{|c|c|}
\hline Images & $\mathrm{NC}$ \\
\hline Image_a & 1.0000 \\
Image_b & 1.0000 \\
Image_c & 1.0000 \\
Image_d & 1.0000 \\
\hline
\end{tabular}

Another possible attack can be the binarization of the grayscale watermarked image. The proposed scheme is also tested against binarization. The values of NC in this case are shown in table 8 .

Table 8: Values of NC under Binarization attack

\begin{tabular}{|c|c|}
\hline Images & NC \\
\hline Image_a & 0.8705 \\
Image_b & 0.9285 \\
Image_c & 0.9071 \\
Image_d & 0.8325 \\
\hline
\end{tabular}

It is obvious from the table 7 that in case of binarization of grayscale watermarked image; the watermark is well able to survive.

\section{CONCLUSIONS}

In this paper, a robust spread spectrum method of digital image watermarking for document and graphics images in wavelet domain is proposed. The algorithm automatically selects the watermark embedding strength depending on amount of details available in image as text or drawing to achieve higher level of robustness. The proposed scheme is tested for various types of images including English Text, Hindi Text, Electronic Circuit Diagram and a Drawing representing wide categories of document and graphics images. The results show that after watermark embedding, there are almost no visible artifacts in the test images thus leaving a clear white background. The robustness of watermarking scheme on example images is also tested under various attacks. Results show that in almost all types of attacks, the proposed scheme survives well and good level of Correlation Coefficient between recovered watermark and original watermark is achieved. In attack cases of noise addition, sharpness and intensity adjustment, the proposed scheme's performance is excellent. Also the binarization of the watermarked image is not able to destroy the watermark. In case of blurring of images, this schemes does not perform so well but such types of attacks are not important as they make the document image unreadable and graphics image useless as in such images, details (sharpness) is important and blurring makes them meaningless.

\section{REFERENCES}

[1] Van Schyndel R. G., Tirkel A. Z., and Osborne C. F., "A Digital Watermark", IEEE Proceedings ICIP, vol.2, pp. 8690, 1994.

[2] Nikolaidis N. and Pitas I., "Robust Image Watermarking in the Spatial Domain", Elsevier, Signal Processing 66, 385403, 1998.

[3] Mukherjee D. P., MaitraSubhamoy, and Scott T. Acton, "Spatial Domain Digital Watermarking of Multimedia Objects for Buyer Authentication", IEEE Transactions on Multimedia, Vol. 6, No. 1, February 2004.

[4] Ganic Emir, Dexter Scott D. and EskiciogluAhmet M., "Embedding Multiple Watermarks in the DFT Domain using Low and High Frequency Bands", Proceedings on Security, Steganography, and Watermarking of Multimedia Contents, Volume VII, vol. 5681, SPIE. 
[5] Solachidis V. andPitas L., "Circularly symmetric watermark embedding in 2-D DFT domain", IEEE Transactions on Image Processing, Vol. 10, 1741-1753, Nov. 2001.

[6] Lin S. D. and Chin Feng Chen, "A robust DCT-based watermarking for copyright protection", IEEE Transactions on Consumer Electronics, Vol. 46, Issue.03, pp. 415-421, August 2000.

[7] Barni M., Bartolini F., Cappellini V.andPiva A., "A DCTdomain system for robust image watermarking", Elsevier, Signal Processing 66, 357-372, 1998.

[8] Hernández Juan R., AmadoMartín and Pérez-González Fernando, "DCT-Domain Watermarking Techniques for Still Images: Detector Performance Analysis and a New Structure", IEEE Transactions on Image Processing, Vol. 9, No. 1, January 2000.

[9] Serdean C. V., Tomlinson M., Wade J. G. and Ambroze M. A., "Protecting Intellectual Rights: Digital WM in the Wavelet Domain", Proceedings of the IEEE International Workshop on Trends and Recent Achievements in Information Technology, Cluj-Napoca, Romania, 16-18 May, 02, ISBN: 973-8335-49-3.

[10] Patvardhan C., Verma A. K. and Lakshmi C. Vasantha, "A Comparative Analysis of Spread Spectrum Watermarking Technique in Wavelet Domain", Journal of Computer Science and Engineering (JCSE), Volume 9, Issue 2, October 2011.

[11] Woo C. S., Du J. and Pham B., "Performance Factors Analysis of a Wavelet-based Watermarking Method", Proceedings of $3^{\text {rd }}$ Australian Information Security Workshop (AISW2005), CRPIT, vol. 44, pp. 89-97, 2005.

[12] Patvardhan C., Verma A. K. and Lakshmi C. Vasantha , "A Robust Wavelet Packet based blind Digital Image Watermarking using HVS characteristics", International Journal of Computer Applications (IJCA), 0975 - 8887, Volume 36, No.09, December 2011.

[13] Brassil J., Low S., Maxemchuk N. and O'Gorman L., "Electronic marking and identification techniques to discourage document copying", IEEE journal of Select. Areas Commun., Vol. 13, pp. 1495-1504, 1995.

[14] Brassil J., Low S., Maxemchuk N. and O'Gorman L., "Copyright Protection for the electronic distribution of text documents", Proceedings of IEEE, no. 07, pp. 1181-1196, 1999.

[15] Mei Q., Wong E. K., and Memon N., "Data Hiding in Binary Text Documents," Proceedings of the SPIE, Security and Watermarking of Multimedia Contents III, vol. 4314, San Jose, California, pp. 369-375, Jan. 2001.
[16] Huijuan Yang and Alex C. Kot, "Data Hiding for Text Document Image Authentication by Connectivity Preserving", ICASSP, IEEE, 2005.

[17] Liu Jin'An, He Lu, Fang DingYi and GuiXiaoLin, "A Text Digital Watermarking for Chinese Word Document", International Symposium on Computer Science and Computational Technology, 2008.

[18] Young-Won Kim and Il-Seok Oh, "Watermarking text document images using edge direction histograms", Elsevier Pattern Recognition Letters-25, 1243-1251, 2004.

[19] Chen M., Wong E. K., Memon N. and Adams S., "Recent developments in document image watermarking and data hiding," in Proc. SPIE Conf. 4518: Multimedia Systems and Applications IV, Aug. 2001, pp. 166-176.

[20] JalilZunera and Mirza Anwar M., "A Review of Digital Watermarking Techniques for Text Documents", International Conference on Information and Multimedia Technology, IEEE, 2009.

[21] Haiping Lu, Xuxia Shi, Shi Y. Q., Kot A. C. and Lihui Chen, "Watermark embedding in DC components of DCT for binary images", IEEE Workshop onMultimedia Signal Processing, $300-303$, Nanyang Technol. Univ., Singapore, 2002.

[22] Hongwei Lu and Wei Xia, "A Robust Binary Image Watermarking Based on Wavelet Domain and Krawtchouk Moments", International Conference on Research Challenges in Computer Science, IEEE, 2009.

[23] FatemehDaraeeand SaeedMozaffari, "Watermarking in Farsi/Arabic binary document images using fractal coding", $8^{\text {th }}$ International Conference on Information Security and Cryptology (ISCISC), September 14-15, 2011.

[24] Daubechies Ingrid, "Ten Lecture on Wavelets", CBMSNSF Regional Conference Series in Applied Mathematics, ISBN: 978-0898712742, 1e, June 1992.

[25] MallatStephane, "A Wavelet Tour of Signal Processing", 3e, 2008, Academic Press, ISBN 978-01-237-4370-1.

[26] Product documentation of Wavelet Toolbox: http://www.mathworks.com/help/toolbox/wavelet/wavelet_ product_page.html.

[27] SuhadHajjara, MoussaAbdallah and AmjadHudaib, "Digital Image Watermarking using Localized Biorthogonal Wavelets", European Journal of Scientific Research, ISSN: 1450-216X, Vol. 26, No. 4, pp.594-608, 2009.

[28] Cox I. J., Kilian Joe, Leighton F. Thomson and ShamoonTalal, "Secure Spread Spectrum Watermarking for Multimedia", IEEE Transactions on Image Processing, Vol. 6, No. 12, December 1997. 